\title{
Necessity, Criteria (Requirements or Limits) and Acknowledgement of Product Identity of Claims for Product Described by Its Manufacturing Process (Product-by-Process Claims)
}

\author{
Kotaro Kageyama \\ Kageyama International Law \& Patent Firm, Tokyo, Japan \\ Email: kageyamalaw@gmail.com
}

Received 15 March 2014; revised 12 April 2014; accepted 4 May 2014

Copyright (C) 2014 by author and Scientific Research Publishing Inc.

This work is licensed under the Creative Commons Attribution International License (CC BY). http://creativecommons.org/licenses/by/4.0/

(c) (i) Open Access

\section{Abstract}

It is inevitably necessary to admit Product-by-Process claims (PBP claims). To make them effective, their requirements and limits should be clarified, and the methods to define the product and acknowledge the identity of the product in their practical exercise should be studied. The author has suggested the following two view points to examine inventions, 1) considering the difficulty to predict an invention from the tentative principles and 2) categorizing inventions into the physicalobject type and the material type. Based on these two viewpoints, this paper will show the case where the PBP claims are inevitable. From the above and its opposite side, the criteria for the PBP claims will be proposed by analyzing the category of inventions, requirements of manufacturing process steps and the limits of admitting the PBP claims. The following theory will be composed of: the scope of the PBP claims should include their identical product to the product described in the claim through the process of the patent applications and infringement cases because of the legal stability. Then, the methods of determining the time to define the product by way of structure or properties and acknowledging the identity of the product, in the practical exercise of the claims will be suggested.

\section{Keywords}

Product-by-Process Claims, Process Limitation Theory \& Identical Product Theory, An Inventions Formed through Experiments, Formation Stages of an Invention, Abbott Laboratories v. Sandoz, Inc., 566 F.3d 1282 (Fed. Cir. 2009) 


\section{Introduction}

In the article titled "The practice of recognizing an inventor/joint inventors and calculation of contribution ratios among joint inventors” (“Joint Inventor Article”) (Kageyama, 2012), I had analyzed the process by which inventions/joint inventions are formed, and discussed and proposed with reference to recognizing the inventor/joint inventors and calculating contribution ratios among joint inventors. In the course of the discussion, I had categorized inventions into physical-object inventions and material inventions (hereinafter these discussion and proposals are “Opinions”).

So far, no discussion on the analysis of inventions such as the above mentioned Opinions is found.

Recently, with reference to product-by-process claims (hereinafter the scope of claims is also called "claims" and product-by-process claims are called "PBP claims"), which "define a product under a product patent by describing the method used to manufacture the same in claims”, their meanings, types, effects and recognition of product identity have become an issue. More specifically, these discussions have been widely carried out earlier, such as (1) whether PBP claims should be recognized solely in cases where it is impossible or difficult to define a product according to its structure or properties, (2) whether claim construction of a PBP claim should be limited to the product produced in the method which is described in the claim ("Process Limitation Theory"), or is admitted to include a product identical to the patented invention even if it is produced by a different process ("Identical Product Theory"), and (3) whether those different theories should be converged to the Identical Product Theory at the phases of examination of application, patent infringement suit, and so forth; or whether the Process Limitation Theory should be adopted in the context of patent infringement suits, as well as those issues specifically discussed in this article, such as (4) timing for defining the product by its structure or properties for the purpose of exercising the patent right and (5) recognition of product identity in the case where the Identical Product Theory is adopted in a patent infringement suit.

USPTO (United States Patent and Trademark Office) and EPO (European Patent Office) present two conditions for recognizing PBP claims: i) the product should have novelty, and ii) it is impossible to define the product other than by way of referring to the manufacturing process thereof.

On the other hand, in Japan, the Intellectual Property (IP) High Court (Grand Panel) rendered a decision on January 27, 2012 (HanreiJiho, 2012) with reference to the patent infringement case ("IP High Court Decision of January 2012”) holding that, where a manufacturing process is recited in a patent claim, (a) the claim should be construed as a "Genuine PBP" claim under the Identical Product Theory if the subject matter product is defined by its manufacturing process, because it is impossible or difficult to define such product directly by its structure or properties (hereinafter this criterion is also called "Impossibility/Difficulty Criteria”), and (b) otherwise the claim should be construed as a "Quasi PBP" claim under the Process Limitation Theory. Further, the court held that the claim construction of Genuine PBP should be made under the Identical Product Theory at the examination phase of the patent.

The present study, based on the Opinions, starts with the discussion on the necessity to recognize PBP claim where it is impossible or difficult to specify such product by its structure or properties (clarification of the aforementioned Impossibility/Difficulty Criteria) and then, on the contrary thereof, proceed to the discussion on the criteria (requirements and limits) for recognizing PBP claims (the "Substantive Issues" of PBP claims so to speak). Then, I argue the definition of product by structure or properties and the criteria for recognizing identity of two products after granting of the patent (the "Actual Effect Issues" of PBP claims so to speak). On the basis of these discussions, I will examine the aforementioned discussions (1)-(5) and make proposals.

The PBP claims, which I recognize under the Opinions, are only limited to those corresponding to the "Genuine PBP Claims” under the aforementioned IP High Court Decision of January 2012, and construed consistently under the Identical Product Theory in the phases of filing an application, patent infringement suit, and so forth.

\section{Abstract of Opinions}

I will briefly explain what I had discussed in the Joint Inventor Article as follows.

\subsection{Opinion 1: Examine the Degree of Difficulty in Predicting an Invention from a Tentative Principle in the Establishment of an Invention (Viewpoint to Examine the Invention from the Process through Which the Invention Was Formed)}

\section{Formation of invention}


An invention is defined as "highly advanced creation of technical ideas utilizing the laws of nature" (Article 2.1 of the Patent Act of Japan).

An invention is formed by (a) conceiving an idea and (b) embodying such conception to achieve certain purpose (or solve a problem). For example, in a case of an invention of airplane, this could be explained as follows.

The laws of nature are typically considered as a principle of physics or chemistry.

In Figure 1, conception based on a principle ("principle") (2. (1) (ii)) and establishment of model ("model") (2. (2) (i)) are important from technical viewpoint and the definition of an invention.

2. Process by which an invention is formed through experiments

There are cases where, although a principle is unknown or difficult to find, the inventions are formed through a discovery of "a relationship where specific results are obtainable under specific experimental conditions" (reproducible phenomenon) in experiments. These cases are described in Figure 2. Experiment is an important method to develop an invention. As discussed later in Paragraph (3.1) 1., the cases under which the use of PBP claim is inevitable are mostly those under which an invention is formed by way of experiments.

An invention is formed when a person conceives a tentative principle, establishes the experimental conditions, and obtains favorable results. The "tentative principle" is a principle which the person believes to be the most appropriate to achieve the purpose at the beginning of the experiments and which also constitutes the basis upon which, one way or the other, the experiments are carried out. However, it is typically rare for favorable results to be obtained in the first try (process). Therefore, based on the results obtained, modifications are made to the previous (conceived) principle, and a new tentative principle is set, based upon which the experimental conditions are set and results are sought.

A tentative principle is generally deemed sufficient even if it is broader than and includes the (true) principle, and even if it is less strict and less accurate than the (true) principle ${ }^{1}$.

3. Classification of the cases where an invention is formed through experiments

This argument would be summarized as follows:

(1) If a principle is difficult to find, and the "tentative principle" (B1) is distinguishable from the "experimental conditions or a product having an effect" (B2: Results of the experiments) when the invention is formed and $\mathrm{B} 2$ are possible to predict* based on the tentative principle, then conceiving the tentative principle is considered to be "conception based on a principle" (including when the tentative principle is the true principle). In this case, setting the experimental conditions (in the case of an invention of a method for producing certain product) and

\begin{tabular}{|c|c|}
\hline $\begin{array}{l}\text { 1. Purpose of invention (problem to be } \\
\text { solved) }\end{array}$ & - I want to fly. \\
\hline $\begin{array}{l}\text { 2. Formation stages of invention } \\
\text { (1) (Proposal of) Conception } \\
\text { (i) Mere intuition } \\
\text { (ii) Conception based on a } \\
\text { principle }\end{array}$ & $\begin{array}{l}\text { - Try to fly like a bird. } \\
\text { - Advance an object and use air resistance to float (use } \\
\text { propulsion to make air resistance and provide lift) }\end{array}$ \\
\hline $\begin{array}{l}\text { (2) Embodiment of conception } \\
\text { (i) Establishment of a model }\end{array}$ & $\begin{array}{l}\text { - Structure: Attach a motor to an object to rotate propellers } \\
\text { and think about the shape of wings }\end{array}$ \\
\hline (ii) Experiments/calculations & $\begin{array}{l}\text { - Experiments: (a) start with parts such as propellers and } \\
\text { wings; (b) model of body; (c) proceed to the full-size body } \\
\text { - Obtain necessary experiment results and formulas }\end{array}$ \\
\hline $\begin{array}{l}\text { (iii) Modification of model } \\
\text { (iv) Completion by repeating } \\
\text { process (i) to (iii) }\end{array}$ & - Object that is practicable \\
\hline
\end{tabular}

Figure 1. Purpose of invention and formation stages of the invention (in the case of inventing an airplane).

\footnotetext{
${ }^{5}$ As an example of a (tentative) principle, in the technology explained in Footnote 5, the principle used in the technology is substantially different, where portion (1) is chemical in terms of improved strength of a substance and portion (2) is physical in terms of balanced weight of load. In an actual invention, for example in case of portion (1), the properties of a product vary depending on the composition of substance, manufacturing process step, and so forth. The basis for producing properties from the composition of substance, manufacturing process step, and so forth would be the tentative principle (see also Figure 5).

Specifically, different tentative principle could be used correspondingly depending on the difference in the composition of substance, manufacturing process step and so forth.

See Footnote 7 regarding the criteria for determining whether two patented inventions "have the same tentative principle in common".
} 


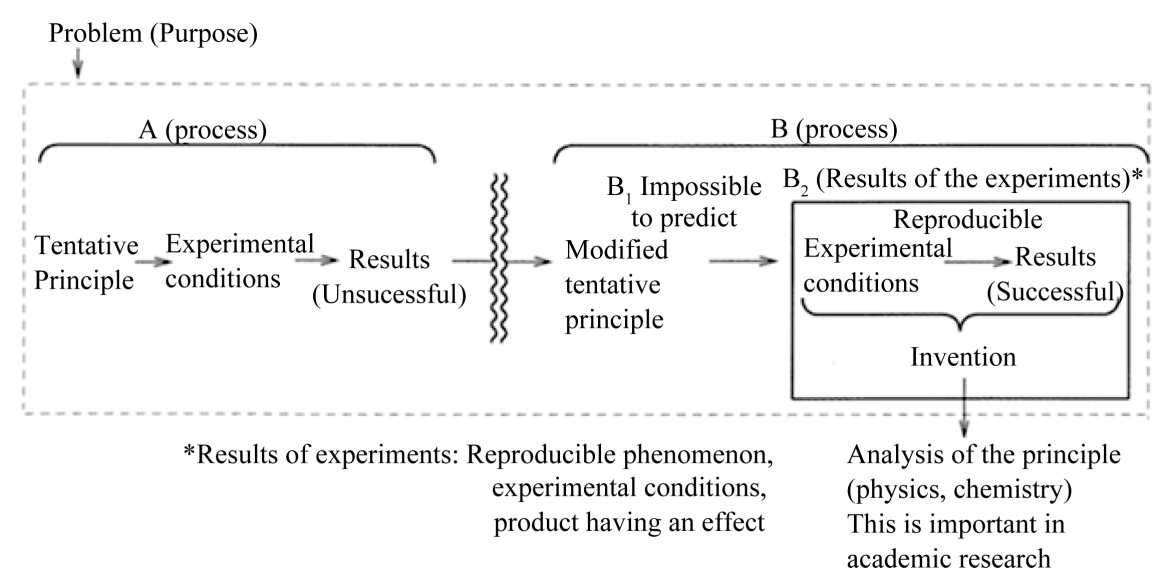

Figure 2. Process through which inventions are formed by experiments (where the experimental conditions and experimental results are not possible to predict based on the tentative principle).

obtaining a product having an effect under those conditions (in the case of a product invention) are deemed to be "establishing a model". Here, the expression "possible to predict" refers to the relative correlation between B1 and B2; technically, the relationship between B1 and B2 is thought to be to an extent where "it can be reasonably explained from theories of physics and chemistry, taking into consideration the technical level at the time of the invention" ( $\mathrm{Y}-2$ of Figure 3).

Correctly, to the extent possible to predict. Hereinafter, the same usage will be applied.

(2) In actual cases, however, there would be many cases where neither the experimental conditions nor a product having an effect are not possible to predict based on the tentative principle. In this case, the conception of a tentative principle is not deemed as a "conception based on a principle", but deemed as a "mere intuition". The "finding and use of reproducible phenomenon" would substitute the conception based on a principle. The reproducible phenomenon, experimental conditions and a product having an effect together appear as an inseparable unit. Even in this case, there could be a principle which is difficult to find, and the principle and model appear as an inseparable unit (Y-1 of Figure 3).

(3) In cases where "the principle is not found (unknown)", it is not possible to set even a tentative principle, as compared with "cases in which the principle is difficult to find" ( $\mathrm{X}$ of Figure 3).

(4) Other cases where an invention is formed through experiments would be those under which the principle is known, but it is difficult to predict a model from the principle, and thus experiments are required. Models (results of the experiments) in such cases can be a product having an effect, device, experimental formula, etc. (Z of Figure 3).

\subsection{Opinion 2: Physical-Object Inventions and Material Inventions (Viewpoint to Examine an Invention from the Way the Invention Appears)}

1. Physical-object inventions and material inventions, and reason for categorizing the types of inventions

A "physical-object invention" focuses on shape of an article, physical structure, or a combination of articles such as circuits. A "material invention" focuses on the nature (material properties) of an article (including its transformation) $)^{2}$. This categorization of types of inventions is considered in terms of the characteristic (key) components of an invention.

In case of a physical-object invention, one can easily recognize the principle and model, and in many cases, the principle is also easy to find, and the principle and model can be divided. Therefore, the process of formation of an invention under (2.1) 1. typically applies. In case of a material invention, on the other hand, the principle is not found or difficult to find in many cases and inventions are made through experiments accordingly. In some

\footnotetext{
${ }^{2}$ For example, when you deal with the problem of insufficient strength of support against load, you can (1) adopt a new material for the support or (2) add new support so that the load is balanced among multiple supports. The method described in (1) is the material type, and the method described in (2) is the physical-object type. Many of the inventions in mechanical fields are the physical-object inventions, and the inventions in chemical fields are the material inventions. In the electrical fields, the inventions related to (a) circuits and the like would be the physical-object type, and those focusing on (b) properties of an article such as semiconductor or magnetic substance would be material type.
} 


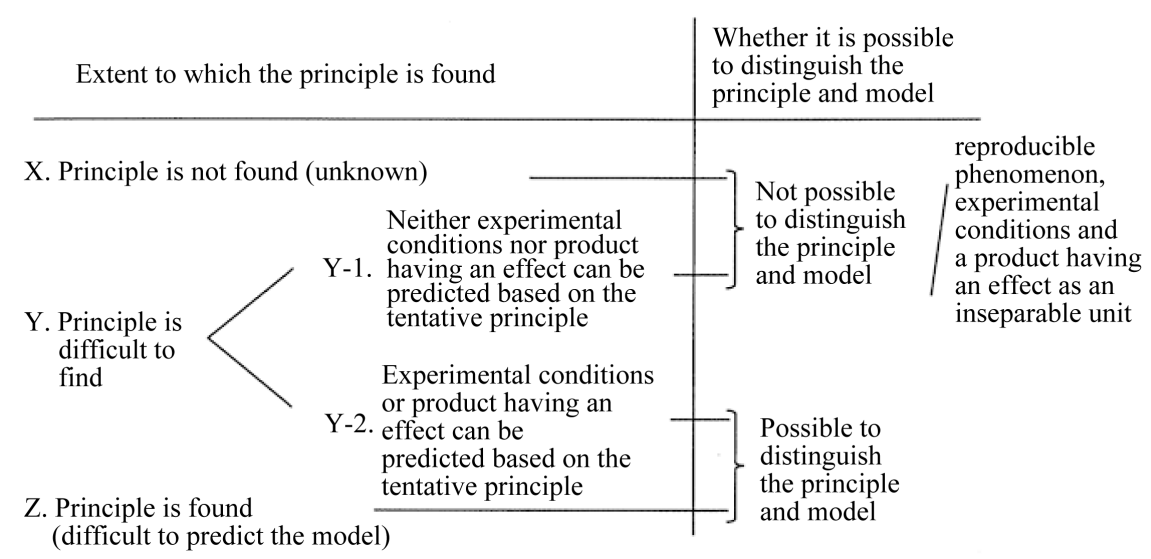

Figure 3. Classification of cases where the invention is formed through experiments.

cases (a), the experimental conditions or a product having an effect are possible to predict based on the tentative principle, and in other cases (b), such prediction is not possible. In the cases of (b), the reproducible phenomenon and the model (experimental conditions or a product having an effect) appear as an inseparable unit, and in many cases, they cannot be distinguished.

\section{Process Inventions}

Process inventions are deemed to "include chronological element". Each chronological step of the process can be considered in the same manner as in the cases of product inventions (whether physical-object type or material type). In the method for manufacturing a product, one may recognize (a) the step to combine articles, etc. and (b) the step under which the properties of the article are transformed among the process steps. Process step (a) is the physical-object element and process step (b) is the material element.

In product inventions also, one can recognize the physical-object process step and material process step in the same manner as the foregoing when one examines the manufacturing method.

\section{Requirements and Limits for Admitting PBP Claims (Substantive Issues on Necessity, Requirements and Limits)}

\subsection{Inevitability of PBP Claims and the Reasons for Such Inevitability}

1. An invention can be formed even if the principle is not found, if one can obtain reproducible and useful product by experiments or otherwise. As long as the purpose of the patent system is to contribute to the progress of industry by promoting inventions (Article 1 of the Patent Act of Japan), it is reasonable to admit such formation of an invention.

Especially in a case where the principle is not found ( $\mathrm{X}$ of Figure 3 ) or is difficult to find, and the results of the experiments are not possible to predict based on the tentative principle ( $\mathrm{Y}-1$ of Figure 3 ) from the process through which an invention was formed, the experimental conditions, reproducible phenomenon and a product having an effect appear simultaneously as an inseparable unit. Among those elements, the experimental conditions and reproducible phenomenon may specifically indicate the manufacturing method of the product. Accordingly, in some cases it is difficult to identify (define) a product by its structure or properties and it becomes inevitable to depend on the manufacturing method to identify (define) the product.

This is the explanation of inevitability, rather than necessity to admit PBP claims ${ }^{3}$.

2. In my opinion, the scientific and technological reason for the foregoing phenomenon is as follows: (1) Scientifically, identification of model by structure or properties of an article is difficult because statically the laws of nature (principle) or dynamically the operation (function) of the laws of nature is difficult to find, and it is also difficult to find scientific technique of analysis to find the same; (2) from technological point of view, use of the manufacturing method to identify an invention is inevitably convenient because the experimental conditions and the reproducible phenomenon, especially the former, are connected to the manufacturing method where the reproducible phenomenon, experimental conditions and a product having an effect appear as an inse-

\footnotetext{
${ }^{3}$ In reality, some of PBP claims consist of the parts that express the manufacturing process (step) and express structure or properties even if insuffi-
} cient to identify a product. 
parable unit.

3. Adding the static scientific viewpoint to discuss the operation of the patent system in more detail, my opinion is as follows:

Generally as a "structure", it is difficult to accurately find up to the atomic arrangement, etc. "Composition" means to see the elements by breaking down an article. Composition is an element to indicate a structure, but does not indicate the state of the article that provides certain function (operation and effect). The terms "characteristics", "properties" and "nature" basically have the same meanings in common, which can be recognized as the way of the appearances of an invention by measurement or other method; for example, by measurement of fusion point, modulus of elasticity, and so forth.

It is difficult to find the chemical structure of an article at issue, to the level of atomic arrangement, etc., both technically and for reason of cost.

As long as the purpose of the patent system is as discussed earlier (Article 1 of the Patent Act of Japan), it would become an obstacle in the operation of the patent system if such a difficult task of finding is imposed on the applicants. Accordingly, in my opinion, the identification of a "structure" would be sufficient as long as the degree of identification is deemed reasonable under the operation of the patent system.

In general, the present study uses the terms "structure", "structure or properties", "structure or characteristic", "properties, etc." in the same way as they are used in terms of identification of an article.

\subsection{Inventions and Requirements for Process Steps Admitted in PBP Claims}

1. Meaning and content of "process step"

Manufacturing process can be described as a series of process steps arranged in order.

(1) The physical-object process step focuses on the shape, physical structure and combination of an article or articles. The subject of each process step remains as a part of a product having an effect in many cases. In these cases, such an article constitutes a part of the product having an effect.

(2) The material process step focuses on the properties of an article (including its transformation). The degree of transformation of the properties which is the subject of each process step may vary. In some cases, the transformation of the properties is caused under the influence of other process step (while, on the other hand, giving effect to other such process step), and in other cases, the transformation occurs without such interaction. Then, the properties of the article are transformed through the ordered process steps and finally the article becomes a product having an effect. The product produced in each process step may change in quality and does not constitute (remain in) a product having an effect in many cases.

2. Composition of invention where a product invention is described by manufacturing method

A product invention may be described by the manufacturing process as follows.

(a) (Raw) materials $\rightarrow$ (b) manufacturing steps $\rightarrow$ (c) product (having an effect)

In the aforementioned description, the relationship between the physical-object/material invention and the physical-object/material process step can be described as follows.

In a physical-object invention, the process step (a) is physical-object type because it focuses mainly on the shape, structure, and so forth of the subject matter of the process step, as the material, and most likely the process step (b) is also physical-object type. In case of a material invention, the process step (a) is material type because it focuses on the properties of the subject matter of the process step, as the raw material, and in many cases the important portion of the process step (b) is material type focusing on the properties of the subject matter and its transformation.

In conclusion, if the invention is a physical-object invention, the process step thereof is also most likely to be physical-object type, and if the invention is a material invention, the important portion of the process step is likely to be of material type.

3. Physical-object and material process steps and inventions (technology)

Considering the types of the process steps (physical-object or material) as discussed in paragraph 1., the physical-object process steps can be stated as more independent, because those process steps are not affected by other process steps, as compared with the material process steps.

As a result, in a physical-object invention consisting of such physical-object process steps, each process step represents the constituent part of the invention, and a mere "juxtaposition" of those process steps may show the composition of the product. However, in case of a material invention, a mere juxtaposition of process steps can- 
not represent the composition of the product.

As mentioned in the foregoing and in paragraph 2. and (2.2) 1. (the property of an invention), a physicalobject invention can generally indicate the structure of a product even if the manufacturing method is considered, and therefore there is no necessity of using a PBP claim. The necessity of using a PBP claim may arise in cases of material inventions.

4. Requirements for process steps admitted in PBP claims

The cases where it is impossible or difficult to identify a product other than by the manufacturing process, such as in cases of PBP claims, should be considered basically when the process step is a material type, and especially when the transformation of properties in the process step is substantial. In other words, PBP claims can be admitted for those process steps ("requirements for process steps" so to speak).

This means that the "chronological element" ((2.2) 2.) in a manufacturing method is substantially construed as the transformation of properties instead of flow of time.

\subsection{Criteria for Admitting PBP Claims}

\section{Limits on admission of PBP claims}

PBP claims may be admitted only when it is impossible or difficult to identify the product through its structure or properties as an invention. Accordingly, in reverse, those cases would constitute the reasonable limit for admitting PBP claims.

Therefore, the PBP claims should be limited to the cases where "the principle is not found (unknown) or difficult to find, and the results of experiments are not possible to predict based on the tentative principle” (X and Y-1 of Figure 3) as an invention (i.e., "Limits on admission of PBP claims”).

2. Criteria for admitting PBP claims

Under the Opinions, only the claims falling under the scope described in the following are admitted in principle as the PBP claims, pursuant to (3.2) 4., (3.3) 1., and so forth.

(1) The product is patentable ${ }^{4}$ (which is the requirement for a patentable invention);

(2) The invention is a material invention;

(3) The characteristic (key) process step of material type should be adopted (requirements for process step); and

(4) The use of PBP claim should be limited to the cases where "the principle is not found (unknown) or difficult to find, and the results of experiments are not possible to predict based on the tentative principle” as an invention (limits for admitting PBP claims).

However, the scope of inventions is quite wide, and accordingly, with regard to (2), (3) and (4) there could be cases where PBP claims are deemed inevitable, allowing exceptions by examining on a case-by-case basis.

Subject to the requirement of (1), the present study clarifies the subject matter in (2), and sets forth the requirements for process step in (3) and the limits for admitting PBP claims in (4), respectively ${ }^{5}$.

Therefore, as the patent would be invalid as long as the article has no patentability, there would be no infringement of the patent. Further, if the claims are not admitted as PBP claims (meaning the "Quasi PBP claim” under the IP High Court Decision of January 2012), the construction of the technical scope of the claim should depend on the Process Limitation Theory.

The Impossibility/Difficulty Criteria under the aforementioned IP High Court Decision is comprehensively set by (i) formally narrowing down the subject of examination because the above-mentioned Items (2) and (3) are determined from objective point of view, (ii) in Item (4), substantially replacing the terms "impossibility" and "difficulty" with "not found", "difficult to find" and "not possible to predict" which are more objective in

\footnotetext{
${ }^{4}$ Unfortunately in Japan, some inventions referred to as "PBP claims" lack patentability of the product itself, but are patented because of the patentability of the manufacturing method. This requirement is added to exclude such patented inventions. If the patentability of an invention is recognized in the manufacturing method like in this case, its patent should be selected as the invention of the manufacturing method (process), not as the product.

${ }^{5}$ As mentioned earlier, I strictly restricted the scope admitted as the PBP claims. In this connection, in Japan, approximately half of the inventions by the claims referred to as "PBP claims" are physical-object type, and in Abbott decision in US described later, some of the PBP claims in the antecedent picked up in Judge Newman's opinions are physical-object type. Therefore, if we can avoid the use of PBP claim for at least these physical-object inventions, the number of PBP claims would be considerably diminished.

The aforementioned criteria was introduced by adding (a) categorization of inventions (physical-object type and material type) and (b) viewpoints of scientific and technological reasonability by means of the analysis of invention formation process based on the Opinions to the grounds for admitting PBP claims, and by exploring the essence of invention especially of material type.
} 
terms of indicating the degree, and (iii) considering each case on an individual basis.

3. Outline of determination whether to admit PBP claims

The above-mentioned discussions can be described in the following arranged manner.

4. Steps in the course of examination of application

Under the examination procedures, as to application using PBP claims because it is impossible or difficult to identify the product through its properties, etc. the Patent Office requires the claims to identify the invention with its properties, etc. (1) if the invention is physical-object type or (2) if the invention is material type and its characteristic process step is not material type, and admit the use of PBP claims ("Genuine PBP Claim"), (3) if the characteristic process step is of material type and it is clarified by the applicant that "the principle is not found or difficult to find, and the results of experiments are not possible to predict" based on the tentative principle, as an invention. (4) When the Patent Office has any "tentative reasonable doubt" in the inevitability of using PBP claim in the application, it should issue a notice of reasons for refusal on the ground of failure to meet the clarity requirement for the statement of the scope of claims, "the invention for which a patent is sought is clear” (Article 36.6, Item (2) of the Patent Act of Japan). (5) The patentability requirements (novelty, inventiveness, etc.) should be examined as for the articles identified in the foregoing steps.

The relationship between the aforesaid (5) and Figure 4 is as follows: Figure 4 describes whether a claim is deemed as the PBP claims under the Opinions ("Genuine PBP claim”), among those referred to as "PBP claims" currently in Japan.

The term "Quasi PBP claim" is used when an invention is revealed in a patent infringement suit and so forth to have been granted as Genuine PBP claim in the aforementioned steps in error.

\subsection{Problems in Case the "Identical Product Theory" Is Adopted and Responses Taken Based on the Opinions}

As mentioned more specifically in Section 6, the Identical Product Theory is applied to PBP claims upon acknowledgement of subject matter (the gist) of invention under the examination phase, etc., whether in the USA, EU (UK, Germany) or in Japan. However, upon construction of technical scope of invention in the course of patent infringement suits, the USA adopts the Process Limitation Theory. This means that a patent with PBP claim can be granted but the effect of the patent is limited by the manufacturing method because the identification of the subject article is insufficient. On the other hand, Germany and Japan adopt the Identical Product Theory, on the ground that the construction of an article should be consistent in the acknowledgment of subject matter of invention and in the construction of technical scope under patent infringement suits, as long as the PBP claims are admitted. I, as the author of the Opinions, assume that the best way would be to establish a theoretical framework and methods to enable such consistent approach.

The problems of (1) identification (definition) of an article by its structure or properties in PBP claims and (2)

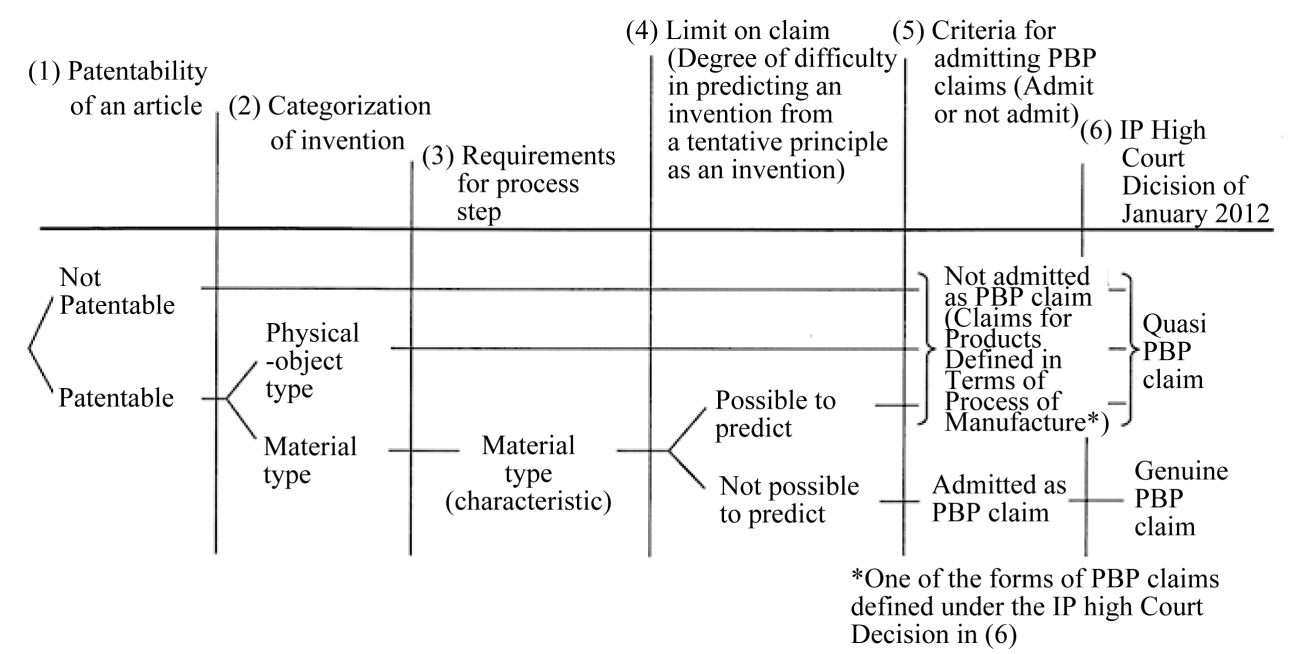

Figure 4. Determination of whether to admit PBP claims. 
acknowledgement of identity of articles, and the problem that (3) publication of an article described by PBP claim remain insufficient, as discussed in Sections 4-6, arise when the Identical Product Theory is adopted in patent infringement suits and so forth (1) and (2) are disadvantageous and (3) is advantageous to the patent owner). Unless the problems of (1) and (2) are solved, one cannot expect any actual effect through exercise of patent, even if the effect of such PBP claims is deemed to include identical products. Therefore, under the theories based on the Opinions, I have conducted reasonable study and tried to clarify the issues to match the aforementioned (1) and (2) directly to the requirements, especially the limits, for admitting PBP claims. For problem (3) as well, the insufficiency of PBP claims is diminished by the reasonable delay in the timing of identification (definition) of an article, indirectly (reflectively) so to speak.

On the other hand, under the Process Limitation Theory, the aforementioned problems (1)-(3) do not arise because the construction of the article described in the claim is limited to the article produced in the process of manufacture, which is the same as described (published) in the claim, and the structure or properties of the article is not examined separately.

\section{Studies on Insufficiency of PBP Claims}

\subsection{Insufficiency of PBP Claims}

PBP clams are "insufficient", so to speak, in the following two respects. First, the effect of patent is economically important in that (a) one can implement their own invention and also (b) can implement the invention by excluding others from implementing the same invention. Under the regular patents, such exclusive implementation is possible from the time one obtains the patent. However, in case of PBP claims, as the structure or properties of the article invented is unclear at the time the patent is granted, it is impossible to compare and examine the identity of the invention and suspected article. Therefore, the patent owner cannot exercise the patent exclusively in implementing the invention, and PBP claims are "insufficient" for the patent owner in that sense.

Second, the publication of the invention is insufficient in terms of the identity of the subject article. Therefore, the patents defined by PBP claims are insufficient as a patent for third parties in that sense.

Later on, when the structure or properties of the article become clear with the progress of technologies, and the article can be defined accordingly by its structure or properties, the effect of exclusivity on the first point would be "sufficient" but the publication of invention on the second point remains "insufficient". In other words, patents defined by PBP claims disclose the invention insufficiently and obtain insufficient benefit of the right until the structure or properties of the article become clear, and after the clarification of the structure or properties, those patents still disclose the invention insufficiently but can obtain sufficient benefit of the right.

Furthermore, if the applicant identifies the invention with its structure or properties only to the extent possible and uses the manufacturing process to identify the invention which cannot be described otherwise at the time when he/she obtains the patent, the applicant (or patent owner) may exercise the effect of exclusivity, because it seems that he/she can find the structure or properties at earlier time in the future than otherwise.

\subsection{Advantages for Patentees Obtained from Insufficiency of PBP Claims and the Adjustments to Be Made for Such Advantages}

1. The owners of patents that are defined by PBP claims have the following advantages, as compared with patents defined by regular claims.

(1) The patent can be obtained earlier than otherwise because the identification of an article by the manufacturing method is deemed sufficient.

In addition to the social and economic advantages of being published as patent owner, a later application in the same technological field may be invalidated after grant of patent on the ground of lack of novelty or others.

(2) The structure or properties of the article are not added to the publication as a part of the claims even after they are found at a later date, and third parties who remain uncertain about infringement of the patent may refrain from entering a new business.

2. However, from the viewpoint of patent owners, (a) there is the necessity to admit those PBP claims, (b) the criteria for admitting PBP claims is strict, (c) there is a social effect of producing a patentable product with patentability (novelty, inventiveness, etc.), and (d) the functions of patent that contributes to the progress of technology by disclosing technology are recognized, even though the description of the technology only depends on 
the manufacturing method.

3. Adjustments

As a method to make adjustments of aforementioned 1. and 2., the advantages of PBP claims may be diminished by making the criteria for clarifying the structure or properties of an article reasonable, and admitting the timing when such PBP claims achieve the criteria to be appropriate.

I will discuss this issue in more detail in Section 5, but the conclusion based on the Opinions would be the cases where "the tentative principle is extracted and the results of the experiments (product having an effect) are possible to predict from such principle" ((5.1) 2. (2) (i)).

It is because as follows. The patent owners are unable to exercise their rights sufficiently because the structure or properties of the article is not clear until such time; but once such time has come, it is thought that a person skilled in the art (see Footnote 7) could predict the structure or properties of the article from the claim describing the manufacturing method and the detailed explanation of the invention, etc. at this point of time, with the progress of technologies.

If we discuss the issue of 1. (2) that the publication remains insufficient, based on the theory of legislation, the structure would be published to the third parties if the law requires correction of claims to describe the structure at least upon exercise of the patent, and such publication continues later on.

\section{Definition of Product by Structure or Properties in PBP Claims; Product Identity under PBP Claims and Proof of Identity (Actual Effect Issues)}

\subsection{Definition of Product by Its Structure or Properties in PBP Claims}

1. The function of definition of product

Definition of product by its structure or properties in PBP claims can be construed to have two functions.

First, it determines the timing upon which the patent becomes exercisable.

At the time of obtaining a patent for an invention which is described by PBP claims, the subject matter of the invention is not defined by its structure or properties. Accordingly, the patent cannot be exercised practically as it is. The patent could be exercisable if and when the definition of the product by its structure or properties becomes possible. In fact, the timing of defining the product would be the timing when the patent owner intends to exercise his/her patent right and determines to make (and believes it to be possible to make) such definition, on the ground of the progress of technologies that made it possible to define the product by its structure or properties.

Second, it provides the grounds for making comparison and confirming identity of two articles.

For example, if the subject matter of the invention can be defined by its structure or properties and the suspected infringing product also can be defined by its structure or properties, it is possible to compare those two products, examine the identity thereof, and determine whether the suspected product infringes the patent.

It is generally difficult to define a product, but in case of inventions described by PBP claims, the definition of the product by its structure or properties is made only after a patent is granted for such invention. Unless the issue of how a product should be defined by its structure or properties is solved, it is actually impossible to allege PBP claims, especially in the course of patent infringement suit, and the admission of PBP claims remains useless.

2. Definition of a product by its structure or properties

(1) The relationship among the structure, principle, properties, and the operation and effect based on the Opinions.

The relationship among the structure, (tentative) principle, properties, and the operation and effect could be described as follows if such description is based on the Opinions and made in terms of social functions and ability of human being (the term "visible" can be treated as one form of "cognizable").

A (tentative) principle is generated by a structure, and properties come from a (tentative) principle. The effect comes from properties.

Under the Opinions, (a) the difference between "structure or properties" and "operation (function) and effect" under judicial precedents is arranged as shown in Figure 5, and (b) one should seek to define the "structure" which is not yet found or difficult to find from the "(tentative) principle", so that the structure could be "conceived" to the extent possible and could have certain position. The "(tentative) principle" is non-cognizable and invisible, but can be conceived. Therefore, I think that the act of seeking for the principle is an extremely human act. 


\begin{tabular}{llll}
$\begin{array}{c}\text { A. Capable of } \\
\text { industrial } \\
\text { application }\end{array}$ & $\begin{array}{c}\text { B. Cognizable } \\
\text { (Visible) }\end{array}$ & $\begin{array}{c}\text { C. Conceivable } \\
\text { (Invisible) }\end{array}$ & $\begin{array}{c}\text { D. Non-conceivable } \\
\text { (Invisible) }\end{array}$ \\
\hline $\begin{array}{l}\text { a. Operation } \\
\text { and effect }\end{array}$ b. Properties & $\begin{array}{l}\text { c. (Tentative) } \\
\text { principle }\end{array}$ & d. Structure \\
"Structure or properties" \\
under Jananese judicial precedent \\
(except for "C" above)
\end{tabular}

Figure 5. The relationship among the structure, (tentative) principle, properties and the effect based on the Opinions.

(2) Theory based on the Opinions regarding definition of a product by its structure or properties.

(i) Definition of a product by its structure or properties

A product should be deemed defined by its structure or properties "if a tentative principle is extracted and the results of the experiments are possible to predict based on the tentative principle" in PBP claims.

(ii) Reason

The reason is that the results of the experiments are deemed to represent the structure or properties of the product in that case. It is assumed that the structure or properties of the product having an effect should be known if the tentative principle and the operation (function) thereof are known (see (3.1) 2. (1)). At this point, the situation is deemed as identical to the situation of "establishing a model" stated in (2.1) 3. (1). In other words, in my opinion, the structure or properties obtained in this way would serve sufficiently as the "structure or properties”.

If "a tentative principle... are possible to predict" as given in (i) at the time obtaining the patent, the PBP claim should not have been admitted as in (3.3) 1. The product should have been deemed as being possible to define by its structure or properties. Then, the case of "a tentative principle... are possible to predict" given in (i) can be deemed as the criteria for admitting PBP claims seen from the opposite side. Therefore, if the situation where "a tentative principle... are possible to predict" is deemed as the content of the "definition", it should be on the ground that the problem of insufficiency of structure in PBP claim is resolved, and should be deemed reasonable.

\subsection{Product Identity under PBP Claims}

1. Review on conventional identity of products under PBP claims

Tokyo District Court rendered a judgment on September 11, 1998 (HanreiJiho,1999) (case involving polyethylene drawn filament) holding that to find the fact of patent infringement, "it is necessary that (1) the suspected product is found to be actually manufactured under the process at issue, or (2) as a prerequisite that the structure or properties of the product which is defined by such process are clarified, the suspected product is recognized to have the identical structure or properties to the product covered by the patent ${ }^{6}$. Then, the infringement was denied on the ground that "the structure or properties of the product which is defined by such process" were not clarified (there have been almost no cases under which an infringement was found).

There are two theories for the identity of products as stated in item (2).

(i) The structure or properties of the respective products must be completely identical (Complete Identity Theory).

(ii) Identity of the operation (function) and effect of those products is adequate (Operation and Effect Theory).

Mr. Masahiro Nanjo, a registered patent attorney of Japan, more specifically explains it to be "structure or properties of a product manufactured by the said manufacturing process, which structure or properties are essen-

\footnotetext{
${ }^{6}$ Originally, the definition of a product by its manufacturing process in PBP claims was admitted because the structure or properties of the product are difficult to find. In the patent infringement cases, it is difficult for patent owners to prove Item (2) in the text. Then, the result would be the same as that obtained under the Process Limitation Theory which limits the scope only to the product obtained by the manufacturing process at issue (Item (1); just the same product).

It comes from the idea that the patent owner should have such risk because he/she would have obtained the patent by defining the product not by its structure or properties but by its manufacturing process (HanreiJiho No. 1700, p. 145; Hanrei Times No. 1017, p. 227 on the decision of Tokyo District Court on September 30, 1999 (acidglycoprotein case).
} 
tial for causing the operation (function) and effect suitable for the purpose of the invention” (Nanjo, 2002).

2. Identity of products under the Opinions

(1) Defect of the Complete Identity Theory and the Operation and Effect Theory, and relationship between those and the theory based on the Opinions

(i) The Complete Identity Theory is ideal, but in reality such identity is impossible to demonstrate.

(ii) There is a risk that the scope under which the identity is acknowledged could be too broad under the Operation and Effect Theory. One of the reasons could be the insufficient elucidation of the operation (function) and the cause-and-effect relationship between the operation (function) and effect. Further, the terms "suitable for the purpose of the invention" and "essential” in Nanjo's theory are unclear.

(iii) The theory based on the Opinion modifies the defect of the Operation and Effect Theory and proposes the following subject to the descriptions in (5.1) 2.

Instead of the "operation (function)" described in the Operation and Effect Theory, the "tentative principle", which narrows the said "operation (function)" by theorizing it physically or chemically, should be adopted. The results of the experiments (experimental conditions and the product having an effect) correspond to the "effect" described in the Operation and Effect Theory. As "structure" is closer to "tentative principle" than the "operation and effect". The "tentative principle” would be reasonable to suggest the "structure” (see Figure 5).

(2) Theory on identity of products on the basis of the Opinions

(i) Criteria for admitting identity

As the "criteria for admitting identity" of two defined products, the products should be deemed identical upon comparison "if they have almost the same tentative principle in common, and the products having an effect which are possible to predict from such tentative principle have almost the same structure or properties".

(ii) Reason

The technical grounds for the said criteria are as follows: (a) a tentative principle is assumed to be extracted from both products; (b) the products have the same extracted tentative principle in common ${ }^{7}$; (c) it is assumed that the structure of the product having an effect would be known when the product having an effect becomes possible to predict based on the tentative principle; (d) it would be reasonable to assume that the structure of products having an effect, which lead to the same tentative principle in common, would be almost identical. Further, (e) the identity of the structure of products having an effect is deemed sufficient as long as such identity can be almost confirmed; however, the identity of the composition should be necessary because the technique of measurement of composition is developed. (f) As the definition of product in the foregoing is based on the tentative principle, it would be appropriate when comparing the identity of two products to consider the tentative principle commonly applicable to those products.

The most important point in the foregoing is that the tentative principle, which was not segregated at the time of obtaining a patent with the use of PBP claims (see X and Y-1 of Figure 3), becomes to be extracted. In other words, in my opinion, the foregoing degree of structure or properties could be sufficient when considering the identity of products. This point is commonly seen in (5.1) 2. (2) setting forth the definition of a product.

The aforementioned criteria is also related to the criteria for admitting PBP claims as discussed in (3.3) 2., and moreover the theory based on the Opinions is a method that can be actually used.

\subsection{Allegation of Definition, Identity and Infringement of Product Based on the Opinions}

With the theory based on the Opinions, I hereby examine the allegation of "definition $\rightarrow$ acknowledgment of identity $\rightarrow$ infringement" under PBP claims, using the following case.

[Case] A party " $X$ " has a patent covering a product " $A$ " defined by the manufacturing process " $\alpha$ ". Another party " $Y$ " manufactures a product " $B$ " with the manufacturing process " $\beta$ ". " $X$ " alleges infringement of its patent against "Y".

\footnotetext{
${ }^{7}$ Whether the products have the same tentative principle in common is determined on the basis of the technical standard at the time of filing of the patent application, namely, at the level of knowledge of a person skilled in the art (a personordinary skilled in the art of the invention). It should be noted here that the term "person skilled in the art" merely represents the level for determining the tentative principle, and the subject to be determined is the "tentative principle".

Even though the tentative principle is hard to understand on an absolute scale, the difference between such principles is easier to understand and, therefore, comparison of tentative principles is assumed to be easier. For example, where a tentative principle comprises several principles, if a new principle is added to such tentative one, or if some of the several principles are changed to others, and consequently the effective results are obtained, it is assumed that such a principle would constitute a different tentative principle.
} 
(1) For "X" to exercise its patent right, the product "A" must be defined by its structure or properties (requirement for exercising patent $)^{8}$.

In other words, the tentative principle of the " $\alpha$ " has been extracted and the structure or properties of " $\mathrm{A}$ " has been predicted from such tentative principle.

(2) " $\mathrm{X}$ " alleges against " $\mathrm{Y}$ " that " $\mathrm{A}$ " and "B" are identical.

(3) "Y" denies the identity of the products and insists that " $\mathrm{B}$ " is manufactured through " $\beta$ ", which is different from " $\alpha$ ".

(4) " $X$ " extracts a tentative principle from the " $\beta$ ".

(5) "X" demonstrates that " $\beta$ " has almost the same tentative principle as " $\alpha$ ".

(6) " $X$ " further demonstrates the structure or properties of " $B$ " which are possible to predict from the tentative principle of " $\beta$ ".

(7) Then, " $X$ " demonstrates that " $\mathrm{B}$ " and " $\mathrm{A}$ " are almost identical. In this case, the identity of composition of "B" and " $A$ " are demonstrated.

(8) "A" and "B" are deemed identical as set forth in the foregoing. Finding of infringement.

In the course of the abovementioned (5)-(7), reasonable (and actual) distribution of burden of proof may be considered. For example, " $X$ " proves the common tentative principle of (5), the structure or properties of the products demonstrated in (6) are also (actually) assumed to be identical, and then regarding (7) " $Y$ " is forced to disprove the identity by demonstrating that the structure or properties of " $\mathrm{B}$ " are different from those of " $\mathrm{A}$ ".

\section{Court Decisions and Examination Practice}

\subsection{Court Decisions and Examination Practice in US}

1. Court decision of patent infringement case in the USA Judgment on Abbott Laboratories v. Sandoz, Inc. (2009).

(1) Conclusion

Process Limitation Theory was adopted in a patent infringement case. The judgment provided a definite opinion by organizing prior cases including the decisions by the US Supreme Court (adopting the Process Limitation Theory) (for example, Macedo, 2009). Macedo's commentary introduces the dissenting opinion discussed in (3) quite specifically in detail.

(2) Outline of judgment (major opinion; (i) (ii)... are written by the author)

(i) Process terms in product-by-process claims serve as limitations in determining infringement.

Product-by-process claims are limited by and defined by the process.

(ii) The policy of the Patent Office in permitting product-by-process type claims to define a patentable product, where necessary...

(iii) Because the inventor chose to claim the product in terms of its process, that definition also governs the enforcement of the bounds of the patent right.

The inventor declines to state any structures or characteristics of this compound ${ }^{9}$.

(iv) How would the courts ascertain that the alleged infringer's compound is really the same as the patented compound? What analytical tools can confirm that the alleged infringer's compound is in fact infringing, other than a comparison of the claimed and accused infringing processes?

(3) Dissenting minority opinions of the court judge and the supplementary opinion towards that of the author

(i) Judge Newman stated as follows:

“The en banc court's 'simple' hypothetical about... is simply irrelevant to the issue we must resolve”. This is not by process written on the claim, but "for example, in the case at bar, comparing the X-ray diffraction patterns and absorption spectra could show that the products are the same, although their exact crystal structure is undefined", and such methodology should be adopted. In response to this suggestion by Judge Newman, the author discusses with reference to the acknowledgment of identity, regarding the subject products, whether the tentative principles are commonly applicable and whether the products having an effect predicted based on such tentative principles are identical, as described in 5 "Definition of Product by Structure or Properties in PBP

\footnotetext{
${ }^{8}$ As a prerequisite above, "X" should prove again that one had to use PBP claims (Genuine PBP Claims) when one obtained the patent. However, it seems that this proof is easier at this stage because of the progress of the analysis of the product.

${ }^{9}$ This portion suggests strong cautiousness against a situation where the inventor tries not to disclose the structure or properties of a chemical composition.
} 
Claims; Product Identity under PBP Claims and Proof of Identity (Actual Effect Issues)”.

Judge Newman further stated:

"It has been an inviolate rule that patent claims are construed the same way for validity and for infringement".

"My colleagues hold that these are product claims for validity, but process claims for infringement. Departure from the rule that forbids such deviation requires sound reason, and fuller exploration...”

(ii) Judge Lowrie stated the following:

"When a product is old, a product-by-process claim cannot be interpreted as a claim to the product made by any means". But "When a product is new and the inventor claims it by a process of preparation, I fail to see why the product-by-process claim should not be interpreted as a product claim..."

(iii) The above minority opinions contain some points in common with my opinion in this study, and my opinion deepens these discussions.

In the case of Abbott decision, the patent should have been made invalid on the ground of lack of novelty, which is the same case of invalidation of patent as the subsequent case in Japan, the IP High Court Decision of January 2012. Therefore, in the future, as held in the IP High Court Decision, it could be possible that the Identical Product Theory be adopted for Genuine PBP claims, without the limitation by the manufacturing method.

2. Relationship with examination practice

The examination practice in the USA follows the Identical Product Theory. The consistency between such Identical Product Theory adopted in the examination practice and the Process Limitation Theory adopted in court decisions is adjusted by the following logic: The patent for the product is granted, but the effect thereof is limited by its manufacturing method because the definition of the product is insufficient. The incentive for making inventions and legal stability are harmonized in this way.

\subsection{Court Decisions and Examination Practice in Japan}

1. Court decision of patent infringement case in Japan

IP High Court Decision of January 2012 (Pravastatin sodium case) (HanreiJiho, 2012).

(1) Meanings and grounds of claims describing the manufacturing process

When the manufacturing process of the product is described in the claims for a product invention, the technical scope of the invention is limited to the product manufactured by such manufacturing process in principle.

However, under circumstances where it is impossible or difficult to define the product directly by its structure or properties, it is admissible to define the product by the manufacturing process thereof in light of the purpose of the patent system, as stipulated in Article 1 of the Patent Act of Japan and so forth.

(2) Types of such claims

Claims describing the manufacturing process are classified into (a) Genuine PBP claims and (b) Quasi PBP claims. The Genuine PBP claims are construed under the Identical Product Theory, and the Quasi PBP claims are construed under the Process Limitation Theory, as discussed in 1 "Introduction",

(3) Burden of proof

The person who alleges the claim to be a Genuine PBP claim should prove that it is "impossible or difficult" to define the product directly by its structure or properties as mentioned earlier. If the person is unable to prove it, the claim should be construed as a Quasi PBP claim under the principle that the claims should be construed as it is described.

In the aforementioned judgment, the court found that the patent is invalid because the product lacks inventiveness. Further, the court held that the claims should be construed under the Process Limitation Theory as Quasi PBP claims (see Figure 4).

2. Relationship with examination practice

The examination standards were set as follows in October 2003: "where it is impossible, difficult or inappropriate for some reason to define the structure of the product which is the subject matter of the invention directly by its properties and so forth, without reference to the manufacturing process thereof, then the product itself may

\footnotetext{
${ }^{10}$ In my opinion, the validity of patent (satisfaction of the patentability requirements), not the technical scope of the patent invention, should be construed by Identical Product Theory different from the abovementioned decision, because PBP claims are with reference to product inventions and the concept of Identical Product Theory and Process Limitation Theory should be limited to the construction of the technical scope.

And, if the degree of unsatisfaction of criteria for admitting PBP claims is large, the patent by the PBP claims should be construed as invalid by reason of the failure to meet the clarity of claims.
} 
be defined by the manufacturing process thereof".

In the current examination standards set in September 2011, the adoption of the Identical Product Theory was clarified.

As mentioned in (3.4), it would be the most reasonable if the claims are construed consistently by the Identical Product Theory from the examination of application to the infringement suit.

\subsection{Court Decisions and Examination Practice in EU (The UK, Germany)}

1. Court decisions of patent infringement cases in the UK and Germany

Construction in infringement cases, which is a problem related to derived right, should be in accordance with laws of respective designating states.

(1) The UK

Judgment on Kirin-Amgen Inc v. Hoechst Marion Roussel Ltd. (2004).

The PBP claims at issue were Claims 19 and 26. The judgment found, according to Paragraph 132 thereof, "I would... revoke the patent on the ground that claim 19 is insufficient... and claim 26 is anticipated", and states that "Hence the patenting of the two product-by-process claims which have failed". Some people argue that this judgment construes PBP claims under the Process Limitation Theory (Grant \& Smyth, 2010). They adopted the Process Limitation Theory, stating that "if a claim contains limiting parameters, why should not the patentee be taken at their word when it comes to deciding the extent of protection? However, as the claims at issue were not acknowledged as PBP claims, it can be assumed that the judgment just presented the scope of claims of common European patents in the UK, rather than the construction of the scope of PBP claims.

In other words, under this UK case, the patent was found invalid just as in the case of the aforementioned IP High Court Decision of January 2012, and the claims at issue should be construed as Quasi PBP claims as used in the said IP High Court Decision. Therefore, this UK case cannot serve as a judicial precedent on whether a Genuine PBP claim should be construed under the Identical Product Theory or the Process Limitation Theory ${ }^{11}$.

(2) Germany

It has been held that "tendency in Germany remains in favour of the general proposition that process terms are not limiting when considering infringement. A recent decision of the Federal Supreme Court (Thermoplastische Zusammensetzung Xa ZR 100/05-25.2.2010) affirms the view that when assessing infringement, process terms in a claim might not limit the scope of protection" (Grant \& Smyth, 2010). Also, Ryuichi Shitara states that "In Europe and Germany, only the Genuine PBP claims are admitted, and the technical scope of the claim and the acknowledgement of content of the claim are construed under the Identical Product Theory” (Shitara, 2013).

2. Examination standards under EPC (European Patent Convention)

The "Guidelines for Examination in the European Patent Office (2012)" provides the following with regard to PBP claims (PART F Chapter IV 4.12).

Claims for products defined in terms of a process of manufacture are allowable only if the products as such fulfill the requirements for patentability. A claim defining a product in terms of a process is to be construed as a claim to the product as such. The burden of proof for an allegedly distinguishing "product-by-process" feature lies with the applicant.

\section{Reasonability of Clarification of Invention and Patent-Related Issues by Opinions}

\subsection{Reason to Enable to Provide Grounds of PBP Claims under the Opinions}

When the structure of the product in the invention is unknown, one of the reasonable methods to clarify such structure would be to analyze and examine from (a) the way the invention, which is cognizable by human being, appears (the external appearance, nature) and (b) the process by which an invention is formed because a human being is involved in the production of such invention. The aforementioned (a) is the viewpoint suggested in Opinion 2, and the aforementioned (b) constitutes the viewpoint suggested in Opinion 1. The key point of Opin-

\footnotetext{
${ }^{11}$ For clarity, the judgment on Kirin-Amgen case stated that “What mattered was the 'essence of the invention' or what we would call the inventive concept" (Paragraphs 22 and 109), and the essence of these "essence of the invention" and "inventive concept" is considered to have the same concept in common as the "(tentative) principle" discussed in this article. The latter is more concrete.
} 
ion 1 is the "(tentative) principle”.

\subsection{Clarification of Patent-Related Issues Concerning the Formation of an Invention, etc. by the Opinions}

The Opinions suggest additional views from technological and natural-scientific aspects for examining patentrelated issues, which had been conventionally examined from social-scientific views only. Further, as mentioned in (7.1), they are reasonable viewpoints to examine the content of inventions from the way in which the invention appears and the process by which the invention is formed.

Therefore, the Opinions may be useful to resolve the patent-related issues other than the issues discussed in this article and the issues of recognizing an inventor/joint inventors and calculation of contribution ratios between joint inventors as discussed in the Joint Inventor Article, especially for the patent-related issues involving the way in which an invention appears, formation of an invention, and content of an invention.

\section{References}

Abbott Laboratories v. Sandoz, Inc. (2009). 566F.3d1282 (Fed.Cir.2009); 90U.S.P.Q. 2d1769.

Grant, G., \& Smyth, D. (2010). The Protection Conferred by Product-by-Process Claim. European Intellectual Property Review, 32, 635-642.

HanreiJiho (1999). No. 1671, 137-144. ("HanreiJiho” and "Hanrei Times” Are the Names of a Japanese Case Law Journal).

Hanrei Times (1999). No. 990, 244-251. (“HanreiJiho” and “Hanrei Times” Are the Names of a Japanese Case Law Journal).

HanreiJiho (2012). No. 2144, 51-80 ("HanreiJiho” and “Hanrei Times” Are the Names of a Japanese Case Law Journal).

Kageyama, K. (2012). The Practice of Recognizing an Inventor/Joint Inventors and Calculation of Contribution Ratios among Joint Inventors. Journal of Intellectual Property Law \& Practice, 7, 590-603. http://dx.doi.org/10.1093/jiplp/jps090

Kirin-Amgen Inc v. Hoechst Marion Roussel Ltd. (2004). UKHL 46. Japanese Translation in “December 2004 edition of European IP News”.

Macedo, C. R. (2009). Infringement of Product-by-Process Claims in US Clarified. Journal of Intellectual Property Law \& Practice, 4, 608-611. http://dx.doi.org/10.1093/jiplp/jpp119

Nanjo, M. (2002). Construction of Rights Based on Product-by-Process Claims. Patent, 55, 21-28.

Shitara, R. (2013). Studies on Acknowledgment of Content and Claim Construction of Product-by-Process Claim-In Response to the Decision of Intellectual Property High Court Special Division on January 27, 2012. Legal Principles and Proposal on Intellectual Properties; Memoirs of Celebration in Honor of Former Judge Toshiaki Makino's Eightieth Birthday. (Nobuhiro Nakayama, Hiroshi Saito, and Toshiaki Imuraed. Seirinshoin), 295. 
Scientific Research Publishing (SCIRP) is one of the largest Open Access journal publishers. It is currently publishing more than 200 open access, online, peer-reviewed journals covering a wide range of academic disciplines. SCIRP serves the worldwide academic communities and contributes to the progress and application of science with its publication.

Other selected journals from SCIRP are listed as below. Submit your manuscript to us via either submit@scirp.org or Online Submission Portal.
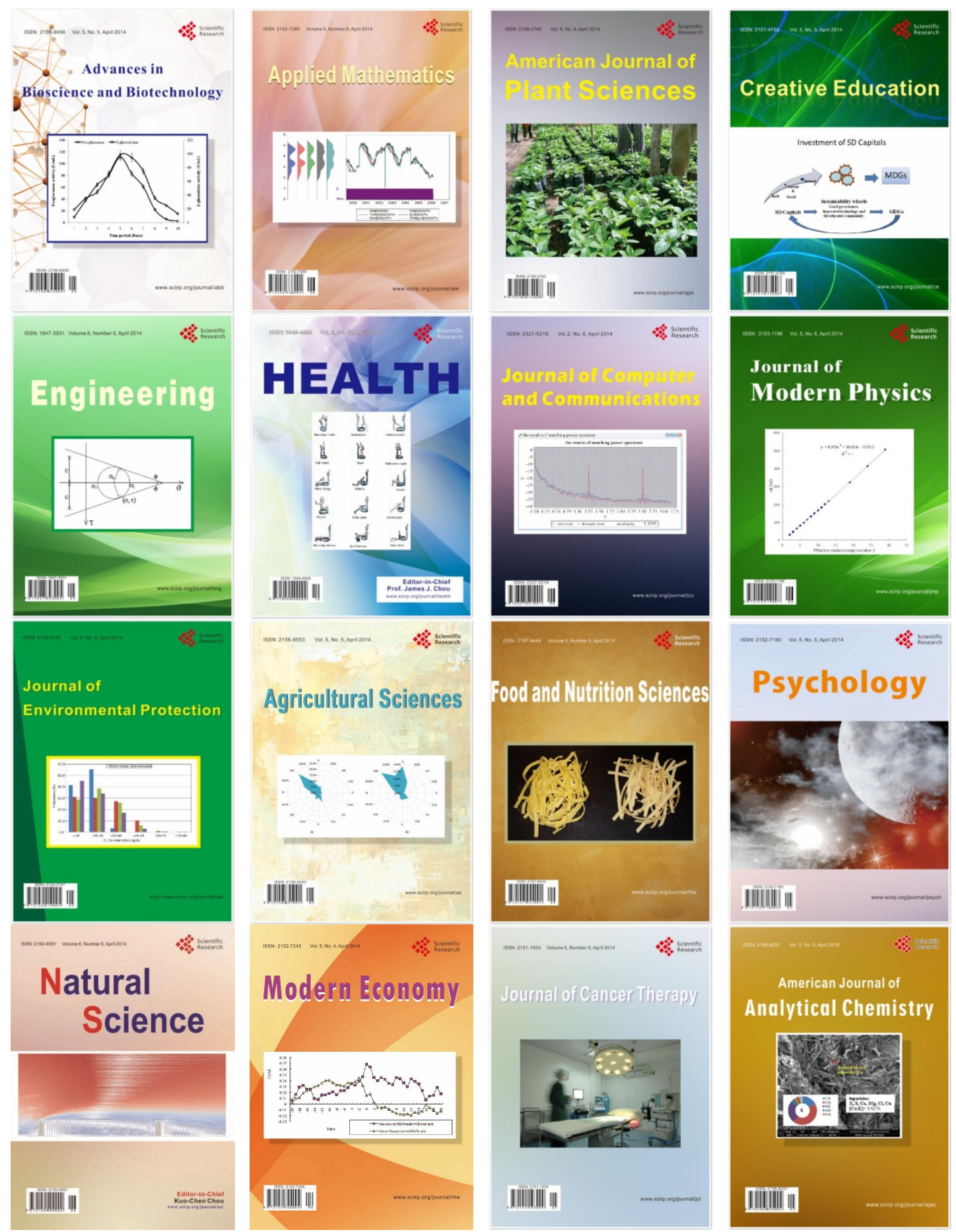